\title{
Indústria e Crescimento: uma Análise de Painel para os Estados Brasileiros*
}

\section{Industry and Growth: a Panel Data Analysis for the Brazilian States}

\author{
Flávio Vilela Vieira** \\ Michele Polline Verissimo*** \\ Ana Paula Macedo de Avellar****
}

\begin{abstract}
Resumo: O objetivo deste trabalho é desenvolver uma investigação empírica para o crescimento do PIB real per capita dos estados brasileiros no período de 1992 a 2009 utilizando dados de painel e estimações por efeitos fixo (EF) e aleatório (EA) e pelo método dos momentos generalizados (GMM). Os resultados econométricos indicam uma relação positiva e significativa entre o PIB da indústria geral e da indústria de transformação, da participação do valor adicionado da indústria geral, mas não da participação do valor adicionado da indústria de transformação para o crescimento econômico dos estados. Além disso, a média dos anos de estudos tem um impacto positivo no crescimento dos estados, e há evidência de que os estados que gastam mais (menos) com custeio e capital têm em média uma menor (maior) taxa de crescimento. Por fim, o desempenho do setor exportador e da inflação nos estados brasileiros não são significativos para explicar as diferenças nas taxas de crescimento.
\end{abstract}

Palavras-chave: Indústria e crescimento. Estados brasileiros. Análise de painel.

Abstract: The main goal of this work is to develop an empirical investigation for the Brazilian States real per capita GDP growth from 1992 to 2009 using panel data and fixed/ random effect and GMM estimation. The econometric results indicate the existence of a positive and significant relation for industry and manufactory industry GDP, the relative participation of the industry in the valued added, but not for the relative participation of the manufactory industry for State GDP growth. The average schooling years have a positive impact on State GDP growth and there is evidence that States with higher (lower) current and capital expenditures in average have lower (higher) GDP growth rates. Finally, State export performance and inflation are not statistically significant to explain differences in growth rates.

Keywords: Industry and economic growth. Brazilian states. Panel data analysis.

JEL Classification: O40; L16; C23.

\footnotetext{
* Os autores agradecem o apoio da Fundação de Amparo à Pesquisa do Estado de Minas Gerais (Fapemig) e do CNPq.

* * Professor do Instituto de Economia da Universidade Federal de Uberlândia (UFU). Pesquisador do CNPq e Fapemig. E-mail: flaviovieira@ufu.br

*** Professora do Instituto de Economia da Universidade Federal de Uberlândia (UFU). E-mail: michele@ie.ufu.br

**** Professora do Instituto de Economia da Universidade Federal de Uberlândia (UFU). Pesquisadora do CNPq e Fapemig. E-mail: anaavellar@ie.ufu.br
} 


\section{Introdução}

O objetivo deste trabalho é investigar empiricamente, através da análise de painel para os estados brasileiros, a relação entre as variáveis PIB da indústria, PIB da indústria de transformação, participação no valor adicionado da indústria e participação no valor adicionado da indústria de transformação, sobre a taxa de crescimento do PIB real per capita estadual no período de 1992 a 2009, controlando também para variáveis já consagradas na literatura teórica e empírica sobre crescimento (nível de renda per capita inicial, inflação, escolaridade média, gastos do governo e exportações).

Parte da literatura sobre crescimento econômico sugere a existência de efeitos favoráveis que o setor industrial e sua participação relativa na economia podem exercer em termos da dinâmica de crescimento nas diversas economias quando comparada aos demais setores (agricultura e serviços), sendo que tais efeitos estão associados a questões como maior dinâmica de incorporação dos avanços tecnológicos e inovações, ganhos de produtividade, maior remuneração dos fatores de produção (capital e trabalho), entre outros.

No Brasil, alguns trabalhos recentes analisam os determinantes do crescimento econômico dos estados brasileiros, como, por exemplo, os efeitos da abertura econômica, dos gastos públicos, da educação e das instituições. Contudo, até o momento poucos estudos foram desenvolvidos para verificar a existência de relação entre a participação do setor industrial e o crescimento econômico dos estados brasileiros, abrindo-se, assim, uma lacuna empírica para a qual este trabalho pretende contribuir.

A investigação empírica deste trabalho é desenvolvida através da estimação de modelos de crescimento com dados em painel e estimações por efeitos fixo $(\mathrm{EF})$ e aleatório (EA) e pelo método dos momentos generalizados (GMM) em diferença, que incorpora a presença da variável dependente (taxa de crescimento econômico) defasada entre as variáveis explicativas.

A estrutura do trabalho envolve mais quatro seções, além desta introdução: a segunda sistematiza uma revisão da literatura sobre indústria e crescimento econômico, englobando alguns estudos para os estados brasileiros; a terceira desenvolve uma análise comparada de indicadores econômicos estaduais selecionados; a quarta realiza uma breve apresentação da metodologia de estimação de modelos em painel e analisa os resultados econométricos; e, para finalizar, a quinta traz as considerações finais da pesquisa. 


\section{Revisão da Literatura: Indústria e Crescimento nos Estados}

Inicialmente, nesta seção se elabora uma breve revisão geral da literatura na qual estão presentes argumentos que destacam a importância do setor industrial para o crescimento e desenvolvimento econômico, antes de se sistematizar os estudos sobre os estados brasileiros.

Parte da literatura sobre crescimento econômico sugere a existência de efeitos favoráveis exercidos pelo setor industrial e sua participação relativa no PIB sobre a taxa de crescimento econômico, sendo que tais efeitos estão associados a elementos que envolvem maior incorporação dos avanços tecnológicos e inovações, ganhos de produtividade, maior remuneração dos fatores de produção e efeitos de transbordamento. ${ }^{1}$ Dentro desse contexto, a estrutura produtiva, e, portanto, a participação relativa do setor industrial, é considerada como um fator importante para se entender as distintas trajetórias de crescimento. A literatura sobre crescimento e indústria discute, ainda, a importância dos investimentos em máquinas e equipamentos (DE LONG; SUMMERS, 1991), sugerindo a existência de uma relação robusta e positiva desses investimentos e o crescimento econômico. Outra dimensão da análise empírica envolve a relação entre indústria, emprego e produtividade (PIEPER, 1998), sendo que as evidências indicam uma relação positiva entre o desempenho industrial e o crescimento econômico, com impactos positivos sobre a geração de emprego e o crescimento da produtividade. Outro aspecto relevante é a questão do grau de diversificação das atividades industriais, especialmente quando se consideram as atividades econômicas intensivas em progresso técnico. O argumento central é de que países cuja estrutura produtiva é predominantemente caracterizada por uma elevada participação dos setores produtivos intensivos em recursos naturais e de baixa intensidade inovadora acabam por ter um pior desempenho ao longo do tempo das taxas de crescimento (COMISIÓN ECONÓMICA PARA AMÉRICA LATINA Y EL CARIBE, 2008; ABELLES; RIVAS, 2010). ${ }^{2}$

Um dos trabalhos mais recentes que relaciona o crescimento econômico aos avanços dos setores de bens comercializáveis e o papel do setor industrial é o de Rodrik (2009), que enfatiza a importância do desenvolvimento das capacidades industriais ao invés de uma estratégia baseada na especialização na produção de

1 Chenery (1960) analisa os padrões de crescimento industrial e deixa claro que um aumento no nível de renda per capita de um país, como indicador de um maior desenvolvimento econômico, está associado a um aumento na participação do setor industrial na produção agregada (PIB). Hirschman (1958) destaca que a estrutura e o desenvolvimento industrial são relevantes para o crescimento econômico através de seus efeitos de encadeamento e externalidades para outros setores. Ver Baer (1972) para uma análise da relação indústria e crescimento em países selecionados da América Latina.

2 Ver Abelles e Rivas (2010) sobre a importância do processo de intensificação da industrialização e seu papel na convergência de renda das economias da América Latina.

VIEIRA, F. V.; VERISSIMO, M. P.; AVELLAR, A. P. M. Indústria e Crescimento: uma Análise... 
bens que contam com vantagens comparativas no comércio internacional. Os resultados empíricos indicam que a expansão da atividade industrial está associada a maiores taxas de crescimento econômico, e uma das lições é de que as mudanças na produtividade das atividades industriais, as transferências de recursos dos setores tradicionais para os setores industriais e a promoção de políticas industriais ativas contribuem para acelerar o crescimento econômico.

Cabe, ainda, destacar análises do crescimento econômico na América Latina, como as de Libânio e Soro (2009), que utilizam o referencial teórico de Kaldor para enfatizar o papel da atividade manufatureira para o crescimento econômico e a relação entre o produto e a produtividade do trabalho na manufatura. Os resultados empíricos indicam a existência de uma relação positiva e direta entre o crescimento do setor manufatureiro e a taxa de crescimento econômico, sendo tal relação associada ao processo de transferência de trabalho dos setores de baixa produtividade para os de produtividade mais elevada, além da existência de significativos retornos de escala na manufatura. Os autores sugerem, ainda, que a possibilidade de ocorrência de ciclos cumulativos de crescimento baseados na expansão das atividades industriais deve ser visto com cautela em contextos marcados pelo aumento da participação de commodities e bens intermediários nas exportações e o declínio nas exportações de manufaturas (desindustrialização) devido aos potenciais efeitos negativos sobre o crescimento no longo prazo. ${ }^{3}$

Alguns trabalhos recentes no Brasil analisam os determinantes do crescimento econômico dos estados brasileiros, tais como os efeitos da abertura econômica, dos gastos públicos, da educação e das instituições. Vale ressaltar que poucos estudos foram desenvolvidos para verificar a existência de relação entre a participação do setor industrial e o crescimento econômico dos estados brasileiros. Desse modo, abre-se uma lacuna empírica para a qual este trabalho deseja contribuir.

Souza (2003) analisa os efeitos da abertura econômica sobre o crescimento dos estados brasileiros, no período de 1991 a 2000, e os efeitos das exportações e importações sobre o PIB e o emprego estaduais. O autor parte da hipótese de que os estados que mais exportam e crescem são os que apresentam vantagens comparativas na produção de algum produto específico. Assim, calcula os coeficientes de vantagens comparativas para os estados brasileiros em relação aos produtos básicos, semimanufaturados e manufaturados. Os estados com vantagens comparativas reveladas em produtos básicos e produtos semimanufaturados foram os que apresentaram maior taxa de crescimento do produto e do emprego no período 1991-2000. O autor avalia que a existência de balança comercial favorável foi um dos principais fatores explicativos do ritmo de crescimento da produção e do emprego da maior parte dos estados. Através da estimação do coeficiente

3 Ver Tregenna (2008), Bresser-Pereira e Marconi (2008), Sampaio e Pereira (2009) e Oreiro e Feijó (2010) para uma discussão mais detalhada sobre desindustrialização no Brasil e na América Latina. 
de Spearman, verifica que a maioria dos estados possui alta correlação entre as exportações e importações com o PIB e o emprego.

Rocha e Giuberti (2007) partem do argumento de que não apenas o tamanho dos gastos públicos afeta o crescimento, mas que a composição desses gastos é relevante, e que certas categorias de gastos favorecem o crescimento econômico, tais como infraestrutura, educação, saúde, defesa, enquanto os gastos com custeio e financeiros impactam negativamente sobre o crescimento. As autoras investigam os efeitos da composição do gasto público sobre o crescimento dos estados brasileiros no período de 1986 a 2002 por meio de análise de painel, com os gastos decompostos em correntes ou de capital e de acordo com sua classificação funcional (defesa, educação, saúde, transporte e comunicação). As evidências apontam uma relação negativa entre a taxa de crescimento e os gastos correntes, enquanto se verifica uma relação positiva entre os gastos de capital e a taxa de crescimento. Ademais, resulta que gastos com defesa, educação, transporte e comunicação afetam positivamente o crescimento.

Ferreira e Ellery Júnior (1996) investigam a existência de convergência entre a renda per capita dos estados brasileiros e calculam a velocidade em que esse processo ocorre. Para isso, o trabalho segue a metodologia de Barro e Sala-i-Martin (1990), estimando uma equação de crescimento (log-linear) derivada da trajetória de transição do modelo neoclássico de crescimento para economias fechadas, em que a convergência seria expressa por uma correlação negativa entre renda inicial e crescimento ( $\beta$-convergência). Além disso, avalia-se a evolução ao longo do tempo da dispersão do produto per capita dos estados ( $\alpha$-convergência). Os autores obtêm resultados que confirmam a hipótese de convergência para os estados brasileiros, mas esta se dá de forma muito lenta. Assim, verifica-se uma tendência dos estados mais pobres reduzirem o gap que os separa dos estados ricos, mas os resultados mostram que são necessários cerca de 50 anos, coeteris paribus, para que essa distância caia à metade.

Dias e Dias (2007) desenvolvem uma análise empírica sobre os efeitos dos investimentos em educação, da distribuição de renda e do nível tecnológico sobre o crescimento da produtividade de médio e longo prazos dos estados brasileiros. O painel desse estudo consiste em dados de 26 estados para um período de cinco anos (1992 a 1996). A metodologia econométrica empregada engloba testes de raiz unitária, heterogeneidade, autocorrelação, dependência seccional e as estimativas de EF. Dentre os resultados encontrados, destaca-se que o aumento do nível educacional dos empregados em relação aos empregadores, as políticas de redistribuição de renda e a tecnologia importada afetam positivamente a taxa de crescimento da produtividade dos estados brasileiros.

Menezes-Filho et al. (2006) investigam o papel das instituições no crescimento dos estados brasileiros. Os autores consideram a existência de relação entre as 
instituições do presente e do passado e buscam estabelecer um vínculo entre elas. Por instituições atuais considera-se o grau de enforcement das leis trabalhistas, e para instituições do passado utilizam-se as seguintes proxies: escravidão, analfabetismo, eleitores e imigração estrangeira. Os resultados obtidos pelas estimações por mínimos quadrados ordinários (MQO) e por variáveis instrumentais apontam que, dentre as variáveis apresentadas, a imigração estrangeira desempenha um importante papel na determinação da qualidade das instituições e, consequentemente, do crescimento dos estados brasileiros. Vale ressaltar, ainda, que a geografia, medida pela latitude das capitais dos estados, mostrou-se mais importante para explicar a heterogeneidade entre as instituições atuais e, portanto, da renda observada na atualidade.

Cruz e Santos (2011) analisam a importância da indústria de transformação no valor adicionado do PIB e no total do emprego para as microrregiões brasileiras. O objetivo desse estudo é verificar a existência do processo de desindustrialização sob a ótica regional, assim é possível verificar se o processo de perda de participação da indústria no PIB tem afetado a distribuição geográfica do emprego industrial. Os autores utilizam dados da Relação Anual de Informações Sociais (Rais) em uma desagregação geográfica por microrregiões para o período de 1990 a 2009. Para medir a concentração do emprego industrial, os autores calculam o índice de Gini, o índice de Moran e o índice de Getis Ord Gi, assim como da metodologia Local indicators of spatial association (Lisa) para identificar aglomerados espaciais. Os resultados encontrados apontam que há desconcentração industrial, sendo que a maioria das microrregiões que mais perderam emprego encontra-se na região Sudeste. Por outro lado, o ganho de importância industrial de algumas microrregiões está condicionado a um maior volume de emprego em indústrias menos avançadas tecnologicamente.

\section{Indicadores Selecionados: Estados Brasileiros}

A Tabela 1 sintetiza o comportamento médio das principais variáveis de interesse deste trabalho: a taxa de crescimento do PIB real per capita dos estados brasileiros, a participação dos produtos estaduais no PIB total do país, a participação da indústria estadual no valor adicionado bruto a preços básicos e a participação da indústria de transformação de cada estado no valor adicionado bruto a preços básicos. 
Tabela 1 - Indicadores de crescimento e da indústria (em \%): estados (média 1995-2010)

\begin{tabular}{|c|c|c|c|c|}
\hline & $\begin{array}{l}\text { Taxa de } \\
\text { crescimento } \\
\text { do PIB real } \\
\text { per capita* }\end{array}$ & $\begin{array}{l}\text { Participação } \\
\text { no PIB }\end{array}$ & $\begin{array}{l}\text { Participação } \\
\text { da indústria } \\
\text { no VAB }\end{array}$ & $\begin{array}{l}\text { Participação } \\
\text { da indústria } \\
\text { de transfor- } \\
\text { mação no } \\
\text { VAB }\end{array}$ \\
\hline Acre & 3,3 & 0,2 & 11,3 & 2,5 \\
\hline Alagoas & 1,6 & 0,7 & 23,7 & 12,1 \\
\hline Amapá & 1,4 & 0,2 & 12,4 & 4,1 \\
\hline Amazonas & 0,1 & 1,5 & 42,0 & 33,4 \\
\hline Bahia & 1,8 & 4,0 & 28,2 & 14,5 \\
\hline Ceará & 2,0 & 2,0 & 23,3 & 12,9 \\
\hline $\begin{array}{l}\text { Distrito } \\
\text { Federal }\end{array}$ & 6,4 & 4,1 & 6,0 & 1,8 \\
\hline Espírito Santo & 2,5 & 2,0 & 31,2 & 15,1 \\
\hline Goiás & 3,4 & 2,3 & 23,9 & 12,4 \\
\hline Maranhão & 4,8 & 1,1 & 16,6 & 6,7 \\
\hline Mato Grosso & 4,7 & 1,4 & 17,7 & 9,6 \\
\hline $\begin{array}{l}\text { Mato Grosso } \\
\text { do Sul }\end{array}$ & 1,7 & 1,0 & 18,2 & 9,2 \\
\hline Minas Gerais & 1,0 & 8,8 & 30,5 & 18,0 \\
\hline Pará & $-0,6$ & 1,7 & 30,8 & 11,6 \\
\hline Paraíba & 2,7 & 0,8 & 21,1 & 10,1 \\
\hline Paraná & 1,5 & 5,9 & 28,3 & 18,1 \\
\hline Pernambuco & 1,3 & 2,3 & 22,1 & 11,5 \\
\hline Piauí & 3,6 & 0,5 & 15,6 & 6,6 \\
\hline $\begin{array}{l}\text { Rio de Ja- } \\
\text { neiro }\end{array}$ & 1,6 & 11,4 & 25,3 & 9,8 \\
\hline $\begin{array}{l}\text { Rio Grande } \\
\text { Norte }\end{array}$ & 2,6 & 0,8 & 23,2 & 7,4 \\
\hline $\begin{array}{l}\text { Rio Grande } \\
\text { do Sul }\end{array}$ & 0,2 & 6,9 & 28,6 & 22,0 \\
\hline Rondônia & 4,2 & 0,5 & 12,5 & 6,7 \\
\hline Roraima & 7,3 & 0,2 & 13,1 & 3,8 \\
\hline
\end{tabular}




\section{Taxa de crescimento do PIB real per capita*}

\section{Participação Participação Participação no PIB da indústria da indústria no VAB de transfor- mação no VAB}

\begin{tabular}{lllll}
\hline Santa & 2,1 & 3,8 & 34,1 & 24,8 \\
Catarina & & & & \\
São Paulo & 1,5 & 34,8 & 30,9 & 22,5 \\
Sergipe & 1,9 & 0,6 & 29,3 & 11,7 \\
Tocantins & 8,2 & 0,4 & 17,0 & 2,9 \\
\hline
\end{tabular}

Fonte: IBGE (2012).

Notas: (*) Dados até 2009; no período de análise deste estudo (1992-2009), ocorreram mudanças na metodologia de cálculo das contas nacionais pelo IBGE nos anos 1997 e 2007, cujo objetivo foi incorporar as recomendações internacionais e atualizar as bases de dados. O conceito de indústria utilizado nesse estudo empírico é o apresentado pelo IBGE (2008) no Sistema de Contas Regionais do Brasil e baseia-se na CNAE 1.0. A atividade industrial consiste na agregação das atividades da indústria extrativa mineral, indústria de transformação, produção e distribuição de eletricidade, gás e água, e esgoto e limpeza urbana e construção civil.

A análise dos dados de taxa de crescimento dos PIBs per capita estaduais revela que os estados com maiores taxas médias de crescimento foram Tocantins (8,2\%), Roraima (7,3\%) e Distrito Federal (6,4\%), enquanto os com menores taxas médias de crescimento foram Pará (-0,6\%), Amazonas (0,1\%) e Rio Grande do Sul $(0,2 \%)$. Percebe-se a ocorrência de uma grande disparidade, no tempo e entre os estados, nas taxas de crescimento, sendo que tais disparidades podem estar associadas a fenômenos populacionais como também em função dos diferentes níveis de desenvolvimento econômico (renda per capita média) entre os estados.

Os dados sobre a participação no PIB dos 27 estados no PIB brasileiro permite destacar que os com maior participação no produto nacional estão concentrados na região Sudeste, sendo que São Paulo $(34,8 \%)$ representa um terço do total, seguido pelo Rio de Janeiro $(11,4 \%)$ e Minas Gerais $(8,8 \%)$. Na sequência, estão os estados da região Sul: Rio Grande do Sul (6,9\%) e Paraná (5,9\%). Os estados com menor participação no PIB brasileiro são Acre, Roraima e Amapá, todos com 0,2\%.

Sobre a participação da indústria no valor adicionado para os estados brasileiros, verifica-se que os estados onde ocorre a maior participação relativa média da indústria são Amazonas (42\%), Espírito Santo (31,2\%), São Paulo (30,9\%), Pará $(30,8 \%)$ e Minas Gerais (30,5\%). Inicialmente, esta análise sugere que esse indicador de participação relativa da indústria não deve ser visto como um indicador de desenvolvimento econômico (renda per capita média), pois os estados com baixo nível de renda per capita (Amazonas e Espírito Santo) estão entre aqueles com 
maior participação relativa da indústria, e não são considerados como representativos do dinamismo industrial brasileiro.

Quando se avalia a participação da indústria de transformação no valor adicionado para os estados brasileiros, percebe-se que os estados onde ocorre a maior participação relativa média da indústria de transformação são Amazonas $(33,4 \%)$, Santa Catarina (24,8\%), São Paulo (22,5\%) e Rio Grande do Sul (22\%). Por conseguinte, a análise do indicador de participação relativa da indústria de transformação, com exceção do Amazonas, indica a ocorrência de maiores valores relativos em estados onde a indústria manufatureira tradicionalmente obteve o seu maior grau de desenvolvimento, ou seja, nas regiões Sudeste e Sul.

De forma sintética, pode-se argumentar que, para o Brasil, o desempenho da participação relativa da indústria geral no valor adicionado tem sido estável nos últimos 16 anos, embora no caso da participação relativa da indústria de transformação se perceba uma redução a partir do auge ocorrido em 2004, passando de $19,2 \%$ para $16,2 \%$ em 2010 .

\section{Modelo Econométrico e Resultados}

O objetivo desta seção é apresentar os modelos estimados por EF ou EA e por GMM em diferença para a taxa de crescimento do PIB real per capita dos estados brasileiros no período de 1992 a 2009.

\subsection{Especificação do Modelo e Estimações Econométricas: Efeito Fixo ou Aleatório e GMM Difference}

Considera-se o seguinte modelo de dados em painel para uma variável dependente $(y)$ :

$$
\mathrm{y}_{i t}=\beta_{1} x_{i t 1}+\beta_{2} x_{i t 2}+\ldots+\beta_{\mathrm{k}} x_{i t k}+\mu_{\mathrm{i}}+u_{i t}
$$

para $t=1,2, \ldots . T$ (dimensão temporal) e $i=1, \ldots . N$ (dimensão cross-section), em que $y$ é a variável dependente, $x$ representa o conjunto de $k$ variáveis explicativas, $\mu_{\mathrm{i}}$ é os EF individuais (específicos a cada estado) e invariantes no tempo e $u_{i t}$ é o termo de erro.

A estimação dos modelos de crescimento com dados em painel deste trabalho é feita inicialmente através dos chamados modelos de EF e EA, métodos utilizados para estimar modelos de efeitos não observados de dados de painel, sendo que a estimação de EF é uma estimação por MQO que usa uma transformação para remover o efeito não observado $\left(\mu_{i}\right)$. A estimação por EA é utilizada quando 
o efeito não observado é não correlacionado com todas as variáveis explicativas e envolve a estimação por mínimos quadrados generalizados (MQG). ${ }^{4}$

Uma limitação dos modelos EF e EA é não lidarem com possível endogenia das variáveis explicativas. Tal limitação é superada através das estimações dos modelos dinâmicos com dados em painel desenvolvidas a partir das estimações GMM.

A estimação de dados em painel dinâmico por GMM está associada aos trabalhos de Arellano e Bond (1991), Arellano e Bover (1995) e Blundell e Bover (1998). A utilização de modelos dinâmicos para dados em painel tem sua justificativa associada ao fato de que muitas séries econômicas se relacionam umas com as outras e com seus valores passados. Nesse sentido, modelos que consideram a variável dependente defasada como sendo uma variável explicativa, no caso de estimações como as de GMM em diferença, distintamente das estimativas em painel estático (EF ou EA), em que ocorre viés nos coeficientes estimados quando se incluem variáveis dependentes defasadas como regressores, podem fornecer estimadores não viesados. A estimação por GMM em diferença para os determinantes do crescimento econômico permite levar em conta a persistência da variável dependente no tempo. ${ }^{5}$

A equação representativa do modelo dinâmico para a taxa de crescimento (y) pode ser assim expressa:

$$
y_{i t}=\delta y_{i, t-1}+X_{i t}^{\prime} \beta+u_{i t}, \text { com } \mathrm{i}=1, \ldots, \mathrm{Net}=1, \ldots, \mathrm{T}
$$

na qual $\delta$ é um escalar; $X_{i t}^{\prime}$ é o vetor de variáveis explicativas $1 \mathrm{x}$ k, que podem ser estritamente exógenas, endógenas ou pré-determinadas; $\beta$ é o vetor de coeficientes $\mathrm{k} \mathrm{x} 1$; e assume-se que o termo $u_{i t}$ segue o seguinte modelo de componente de erro:

$$
u_{i t}=\mu_{\mathrm{i}}+v_{i t}
$$

em que $\mu_{i} \sim \operatorname{IID}\left(0, \sigma_{\mu}^{2}\right)$ e $\sim \operatorname{IID}\left(0, \sigma_{v}^{2}\right)$.

O componente $\mu_{i}$ são os EF individuais (específicos a cada estado) e invariantes no tempo, enquanto $v_{\text {it }}$ representa os choques específicos a cada estado e que variam no tempo, sendo que $v_{i t}$ são heteroscedásticos e correlacionados no tempo. Assume-se, então, que

$4 \quad$ O teste de Hausman fornece a informação de qual dos dois modelos deve ser o selecionado, sendo que a hipótese nula é de que os estimadores por EA são consistentes.

5 As estimações GMM utilizadas neste trabalho foram feitas com o GMM em diferença dado que, em função da dimensão cross-section restrita (26 estados), as estimações GMM system, especialmente para modelos menos parcimoniosos, resultaram em problemas de excesso de instrumentos ou de não validade do conjunto de instrumentos, mesmo após a utilização dos comandos collapse ou laglimits no Stata 12. 


$$
\begin{aligned}
& E\left(\mu_{i}\right)=E\left(v_{i t}\right)=E\left(\mu_{i} \cdot v_{i t}\right)=0 \\
& E\left(v_{i t} \cdot v_{j s}\right)=0 \text { para cada } i, j, t, s \text { com } i \neq j
\end{aligned}
$$

O estimador do GMM de Arellano e Bond (1991) instrumentaliza as variáveis explicativas em diferença $\left(\Delta x_{i t}^{\prime}\right)$ que não são estritamente exógenas com suas defasagens disponíveis em nível. Contudo, nesse estimador GMM de primeira diferença, as defasagens em nível disponíveis podem ser instrumentos fracos para as variáveis não estritamente exógenas caso essas defasagens possam ser caracterizadas como um passeio aleatório. ${ }^{6}$

O objetivo central da investigação econométrica dos modelos de crescimento para os estados brasileiros é avaliar o sinal e a significância estatística dos coeficientes das variáveis PIB da indústria (LPIBIND), PIB da indústria de transformação (LPIBINDTRANSF), participação no valor adicionado da indústria (LINDVAD) e participação no valor adicionado da indústria de transformação (LINDTRANSFVAD), utilizando-se de modelos de crescimento estáticos (EF ou EA) e dinâmicos (GMM em diferença). Com isso, pretende-se avaliar a importância da indústria e da indústria de transformação, tanto em termos de produção (PIB), quanto de seu valor adicionado no crescimento econômico de longo prazo. Os modelos estimados incluem, ainda, outras variáveis consagradas pela literatura empírica de crescimento, como nível de renda per capita inicial (LPIBINICIAL), taxa de inflação (LINF), escolaridade média (LEDUC), gastos públicos com custeio e capital (LCUSTCAPPIB), exportações totais (LEXPORTPIB) e exportações de manufaturados (LEXPORTMANUFPIB). ${ }^{7}$

A estimação dos modelos de crescimento é dividida em dois métodos de estimação. (Ver seção 4.2 para as Tabelas 2 e 3 que reportam as estimações por EF ou EA, enquanto as Tabelas 4 e 5 sumarizam os resultados para as estimações por GMM em diferença). As comparações de sinal, magnitude e significância estatística para os modelos com mesmas especificações, com a exceção de que nas estimações por GMM em diferença foi incluída a variável dependente defasada, porém com métodos de estimação distintos, podem ser feitas a partir da análise comparativa dos resultados entre as Tabelas 2 e 4, e 3 e 5.

As estimações dos modelos foram realizadas com dados de média trienal para o período de 1992 a 2009 (seis observações), com exceção da variável LPIBINICIAL, para a qual se utilizou o PIB per capita inicial em cada um anos iniciais do triênio. O foco da investigação empírica, como já mencionado, é avaliar o sinal,

$6 \quad$ As Tabelas 4 e 5 reportam a probabilidade do teste de Hansen de avaliar a validade do conjunto de instrumentos utilizados nas estimações GMM em diferença, sendo que a hipótese nula é de que o conjunto de instrumentos é válido.

7 Ver o Quadro 1, no Apêndice A, para a lista de variáveis, nomenclatura e fonte dos dados.

VIEIRA, F. V.; VERISSIMO, M. P.; AVELLAR, A. P. M. Indústria e Crescimento: uma Análise... 
a significância estatística e a magnitude dos coeficientes estimados para LPIBIND, LPIBINDTRANSF, LINDVAD e LINDTRANSFVAD. Dessa forma, as estimações podem ser classificadas em um conjunto de quatro classes ( $a, b, c$ e $d$ ) de modelos, nas quais a variável dependente é a taxa de crescimento do PIB real per capita dos estados brasileiros:

a) modelos com LPIBIND (modelos 1, 2, 3 e 4): Tabelas 2 (EF ou EA) e 4 (GMM em diferença);

b) modelos com LPIBINDTRANSF (modelos 5, 6, 7 e 8): Tabelas 2 (EF ou EA) e 4 (GMM em diferença);

c) modelos com LINDVAD (modelos 1, 2, 3 e 4): Tabelas 3 (EF ou EA) e 5 (GMM em diferença);

d) modelos com LINDTRANSFVAD (modelos 5, 6, 7 e 8): Tabelas 3 (EF ou EA) e 5 (GMM em diferença).

\subsection{Resultados Econométricos}

As estimações dos modelos de crescimento são apresentadas nas Tabelas 2, 3 , 4 e 5 para o período de 1992 a 2009 com dados trienais. ${ }^{8}$ Os resultados das Tabelas 2 e 3, na sequência, sumarizam as evidências empíricas para as estimações por EF ou EA dos modelos de crescimento do PIB real per capita dos estados brasileiros. ${ }^{9}$

Os resultados das estimações apresentadas na Tabela 2 revelam que os coeficientes da variável PIB da indústria (LPIBIND) são positivos e estatisticamente significativos em todos os modelos estimados, com coeficientes médios estimados de 5,35, indicando que uma variação de $1 \%$ no PIB da indústria tem um impacto médio de $1,78 \%$ ao ano no crescimento. As estimações para os coeficientes do PIB da indústria de transformação (LPIBINDTRANSF) são positivos, com exceção da estimação do modelo 5 por EA, mas, com exceção do modelo 5, os demais não são estatisticamente significativas. ${ }^{10}$ Então, pode-se argumentar que, diferentemente do PIB da indústria, não há evidência da importância do PIB da indústria de transformação na taxa de crescimento dos estados brasileiros com base nos modelos de EF ou EA.

8 As estimações dos modelos GMM em diferença são GMM 2-step com erros robustos e lidam com o problema da proliferação dos instrumentos. Além da estimação dos coeficientes, foram realizados também os testes de ausência de autocorrelação de segunda ordem, AR(2), e o teste de Hansen para as restrições sobreidentificadoras (validade dos instrumentos).

As estimações finais dos modelos de crescimento (Tabelas 2 a 5) não incluem o Distrito Federal (DF) em função das suas especificidades de ordem geográfica (área), demográfica, de nível de renda per capita e, especialmente, em função da elevada participação do setor público na economia do DF. Os resultados da estimação dos modelos incluindo o DF podem ser solicitados junto aos autores. Em geral, não houve alterações significativas nos resultados sobre o papel da indústria no crescimento dos estados ao se comparar as estimações das duas amostras.

10 A probabilidade do teste de Hausman reportadas nas Tabelas 2 e 3 indica que, dos oito modelos estimados, sete foram por EF) e apenas o modelo 5 foi estimado por EA. 


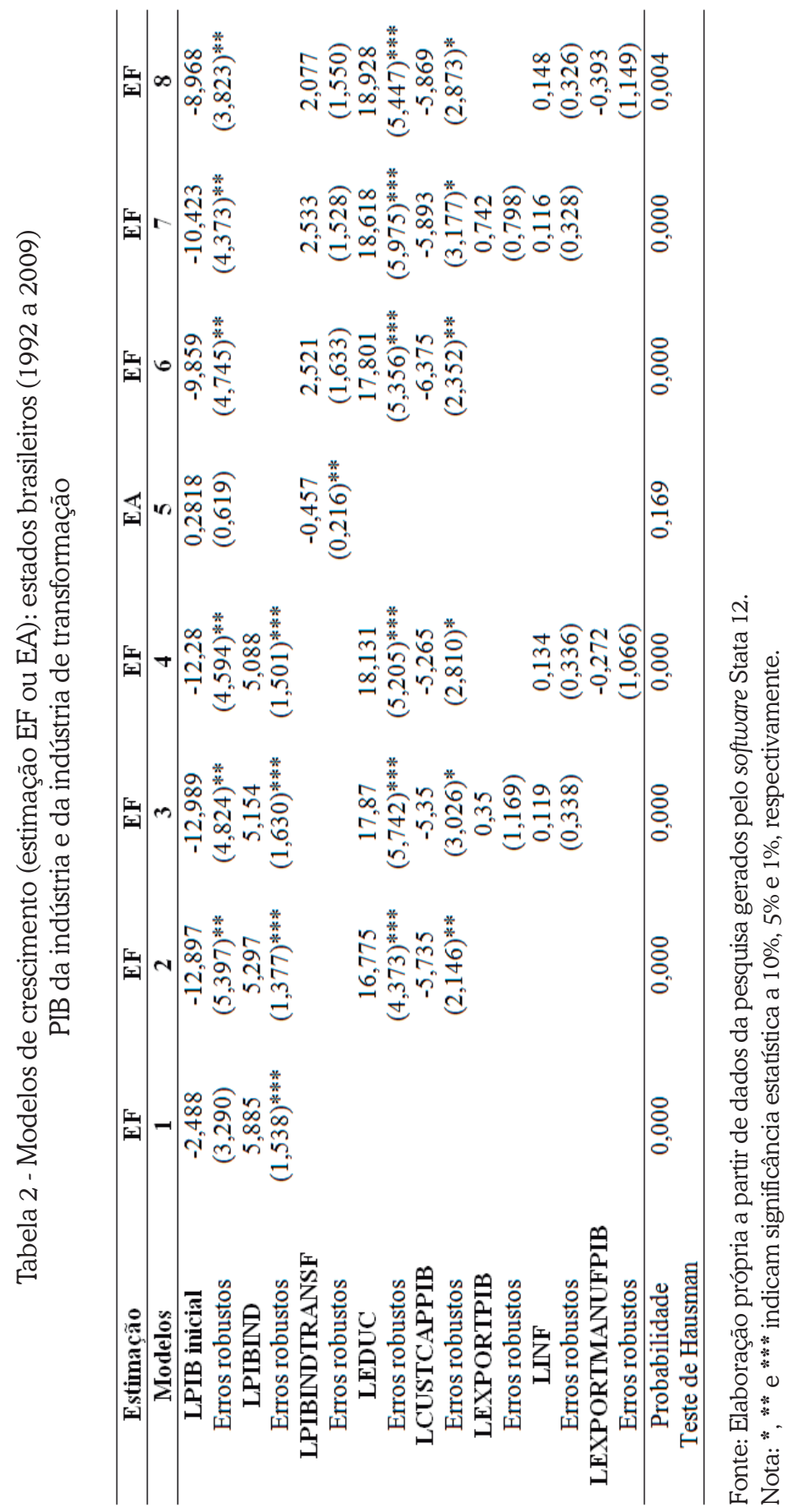

VIEIRA, F. V.; VERISSIMO, M. P.; AVELLAR, A. P. M. Indústria e Crescimento: uma Análise... 
Os dados da Tabela 2 revelam, ainda, que o coeficiente estimado do PIB inicial é negativo em sete dos oito modelos estimados, com exceção do modelo 5 por EA, e estatisticamente significativo em seis dos oito modelos, com exceção dos modelos 1 e 5. Nesse caso, pode-se argumentar que há evidência de convergência de renda entre os estados brasileiros nas estimações por $\mathrm{EF}$ quando utilizadas as variáveis de PIB da indústria e da indústria de transformação. Duas outras variáveis se mostram relevantes como determinantes da taxa de crescimento econômico. Uma delas é a média dos anos de estudo (LEDUC) cujas estimações foram todas positivas e estatisticamente significativas, enquanto os coeficientes estimados da variável gastos com custeio e capital como porcentagem do PIB (LCUSTCAPPIB) se revelam negativos e significativos em todos os modelos estimados. As demais variáveis dos modelos estimados, as exportações totais dos estados como porcentagem do PIB (LEXPORTPIB), as exportações de manufaturados dos estados como porcentagem do PIB (LEXPORTMANUFATPIB) e a taxa de inflação (LINF) não se mostram estatisticamente significativas no modelo de crescimento dos estados brasileiros.

As estimações apresentadas na Tabela 3 revelam que os coeficientes da variável participação do valor adicionado da indústria (LINDVAD) são positivos em todos os modelos e estatisticamente significativos em três dos quatro modelos estimados, com coeficientes médios estimados de 5,01, indicando que uma variação de $1 \%$ na participação da indústria no valor adicionado tem um impacto médio de $1,67 \%$ ao ano na taxa de crescimento dos estados. As estimações para os coeficientes da participação do valor adicionado da indústria de transformação (LINDTRANSFVAD) se alteram de sinais, sendo negativos, nos modelos 5, 6 e 7, e positivo, no modelo 8, e estatisticamente significativo apenas no modelo 5. Desse modo, pode-se argumentar que, diferentemente da participação do valor adicionado da indústria, não há evidência da importância da participação do valor adicionado da indústria de transformação na taxa de crescimento dos estados com base nos modelos de EF ou EA. 


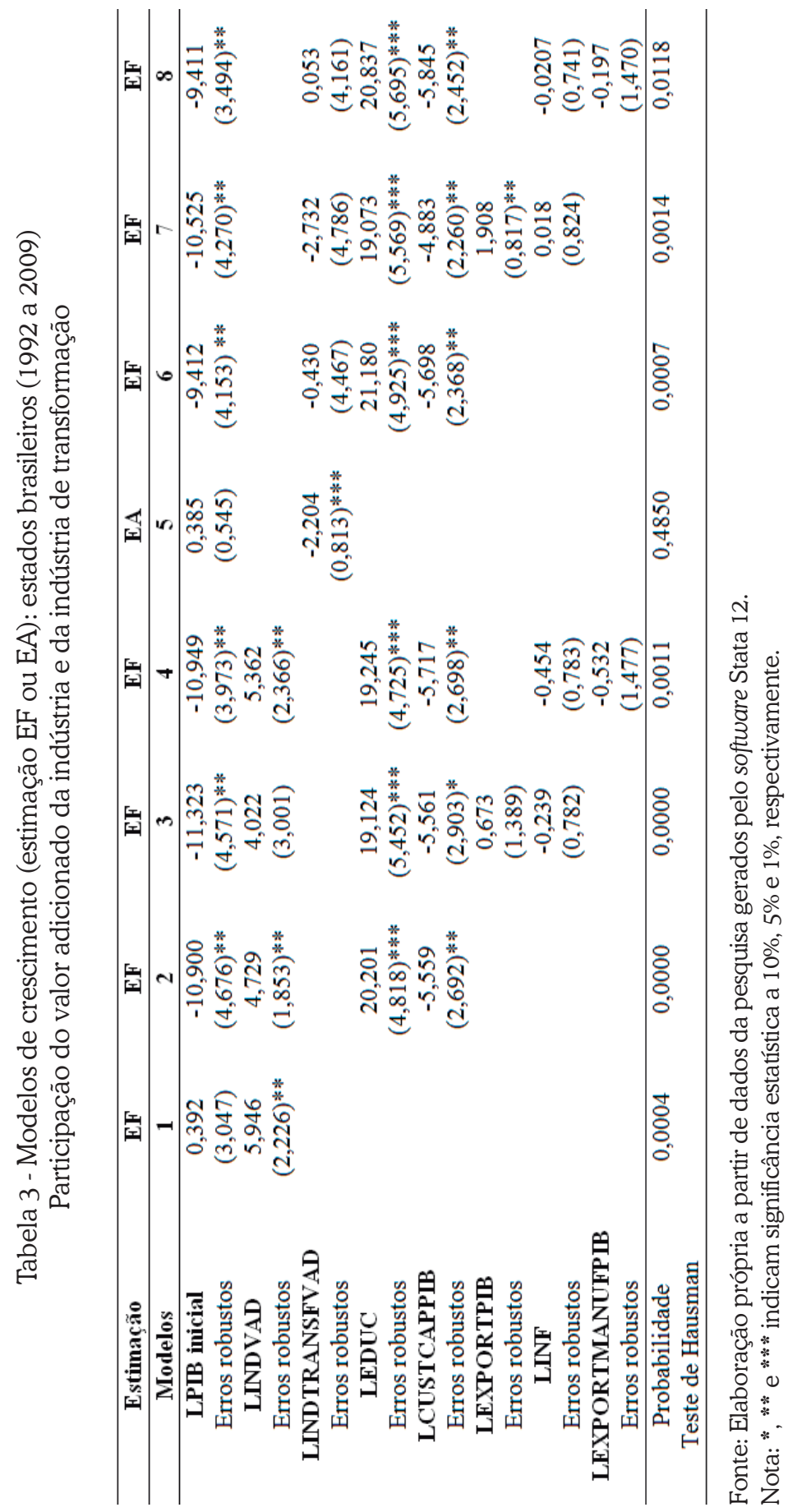


A análise dos demais resultados reportados na Tabela 3 é similar aos já descritos na Tabela 2, com destaque para a significância estatística da variável média dos anos de estudo (LEDUC), com coeficientes estimados positivos e significativos em todos os modelos, gastos com custeio e capital como porcentagem do PIB (LCUSTCAPPIB), com coeficientes estimados negativos e significativos em todos os modelos estimados, e PIB inicial, com coeficientes estimados negativos e significativos em seis dos oito modelos. Quanto às demais variáveis dos modelos estimados, as exportações totais dos estados como porcentagem do PIB (LEXPORTPIB) possui coeficiente positivo nos dois modelos estimados e significativo apenas no modelo 7, enquanto as exportações de manufaturados dos estados como porcentagem do PIB (LEXPORTMANUFATPIB) e a taxa de inflação (LINF) não se mostram estatisticamente significativas no modelo de crescimento dos estados.

Os resultados das Tabelas 4 e 5 sumarizam as evidências empíricas para as estimações por GMM em diferença dos modelos de crescimento do PIB real per capita dos estados brasileiros. Tais tabelas informam, ainda, o uso dos comandos collapse ou laglimits (do Stata 12) para lidar com a proliferação de instrumentos ocorrida em todos os modelos estimados devido ao número restrito de estados (26). ${ }^{11}$

A Tabela 4 revela que os coeficientes da variável PIB da indústria (LPIBIND) são positivos e estatisticamente significativos em todos os modelos estimados, com coeficientes médios estimados de 10,74, indicando que uma variação de $1 \%$ no PIB da indústria tem um impacto médio de 3,58\% ao ano na taxa de crescimento. As estimações para os coeficientes do PIB da indústria de transformação (LPIBINDTRANSF) são positivos e estatisticamente significativos em três dos quatro modelos estimados. Considerando-se a média dos coeficientes estatisticamente significativos, tem-se uma magnitude de 4,40, indicando que $1 \%$ de variação do PIB da indústria de transformação está associada a um impacto médio de 1,47\% ao ano na taxa de crescimento dos estados brasileiros. Cabe ressaltar que as magnitudes dos coeficientes estimados para LPIBIND e LPIBINDTRANSF são maiores nas estimações por GMM em diferença quando comparadas com as estimações por EF ou EA (Tabela 2).

Os resultados da Tabela 4 revelam, ainda, que o coeficiente estimado para o PIB inicial é negativo em seis dos oito modelos estimados e significativo em cinco desses modelos. Assim, pode-se argumentar que há evidência de convergência de renda entre os estados nas estimações por GMM em diferença quando utilizadas as variáveis de PIB da indústria e da indústria de transformação, com exceção nos casos dos modelos mais parcimoniosos (1 e 5), que envolvem problemas de omissão

11 Ver Roodman (2009a, 2009b) para maiores detalhes de como estimar os modelos GMM em painel através do comando xtabond 2 do Stata 12, além das consequências da proliferação de instrumentos. Nesse sentido, foram utilizados os comandos collapse ou laglimits para lidar com o excesso de instrumentos. Ver Windmeijer (2005) para a discussão sobre a correção em amostras finitas na matriz de covariância de forma a tornar as estimações 2-step com erros robustos mais eficientes. 
de variáveis relevantes, o que não acontece com os demais modelos estimados. Assim como nas estimações por EF ou EA (Tabela 2), os coeficientes estimados da variável média dos anos de estudo (LEDUC) são todos positivos e estatisticamente significativos, com magnitude dos coeficientes estimados maior do que a obtida nas estimações da Tabela 2. As estimativas dos coeficientes da variável gastos com custeio e capital como porcentagem do PIB (LCUSTCAPPIB) se revelam negativos em todos os modelos estimados, com significância estatística em três dos seis modelos. Ou seja, tais resultados são menos robustos em termos de significância estatística quando comparados aos obtidos nas estimações por EF ou EA (Tabela 2), mas ainda assim há evidência de que os estados que possuem maiores (menores) gastos com custeio e capital crescem em média menos (mais). Os coeficientes estimados para as variáveis exportações totais dos estados como porcentagem do PIB (LEXPORTPIB), as exportações de manufaturados dos estados como porcentagem PIB (LEXPORTMANUFATPIB) e a taxa de inflação (LINF) não se mostram estatisticamente significativos.

Os testes de ausência de autocorrelação de segunda ordem, AR(2), e de validade dos instrumentos (Hansen) reportados na Tabela 4 indicam não rejeição da hipótese nula em todos os modelos estimados, ou seja, os modelos estimados não possuem autocorrelação de segunda ordem, e o conjunto dos instrumentos é válido. 


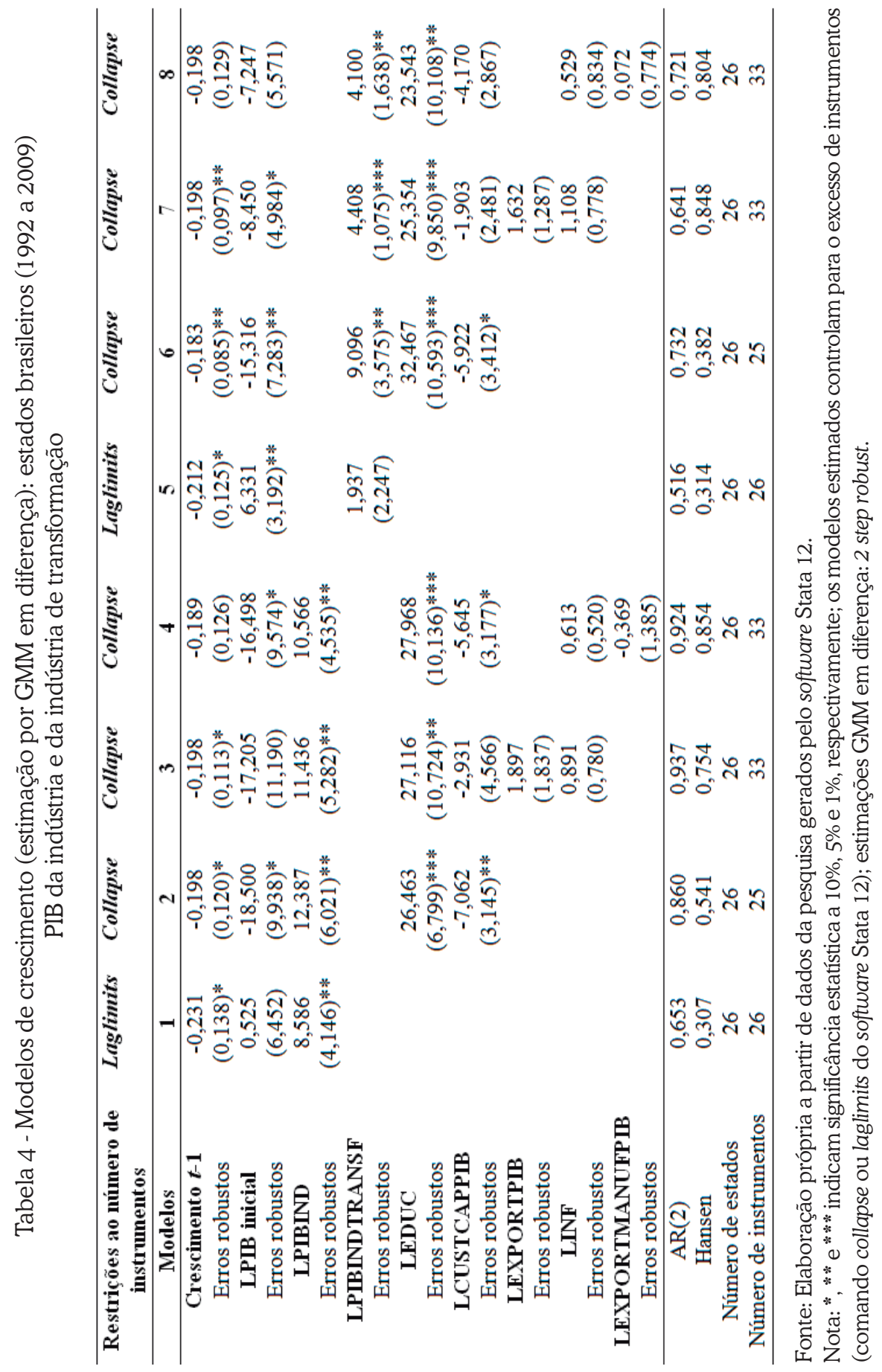


As estimações apresentadas na Tabela 5 revelam que os coeficientes da variável participação da indústria no valor adicionado (LINDVAD) são positivos e estatisticamente significativos em todos os modelos estimados, com coeficientes médios estimados de 14,16 , indicando que uma variação de $1 \%$ na participação da indústria no valor adicionado tem um impacto médio de $4,72 \%$ ao ano na taxa de crescimento. As estimações para os coeficientes da participação da indústria de transformação no valor adicionado (LINDTRANSFVAD) não se mostram estatisticamente significativas nos quatro modelos estimados. Cabe ressaltar que as magnitudes dos coeficientes estimados para LINDVAD são maiores nas estimações por GMM em diferença quando comparadas com as estimações por EF ou EA (Tabela 3).

Os resultados da Tabela 5 revelam, ainda, que o coeficiente estimado para o PIB inicial é negativo em seis dos oito modelos estimados, sendo estatisticamente significativo em apenas no modelo 7 e com o sinal positivo. Nesse caso, pode-se argumentar que não há evidência de convergência de renda entre os estados brasileiros nas estimações por GMM em diferença quando utilizadas as variáveis participação da indústria e da indústria de transformação no valor adicionado. Assim como nas estimações por EF ou EA (Tabela 3), os coeficientes estimados da variável média dos anos de estudo (LEDUC) são todos positivos e estatisticamente significativos. As estimativas dos coeficientes da variável gastos com custeio e capital como porcentagem do PIB (LCUSTCAPPIB) se revelam negativas em todos os modelos estimados, sendo que em quatro dos seis modelos verificou-se significância estatística. Ou seja, tais resultados são menos robustos em termos de significância estatística quando comparados aos obtidos nas estimações por EF ou EA (Tabela 3), mas ainda assim há evidência de que os estados que possuem maiores (menores) gastos com custeio e capital crescem em média menos (mais). Os coeficientes estimados para as variáveis exportações totais como porcentagem do PIB (LEXPORTPIB) e a taxa de inflação (LINF) não se mostram estatisticamente significativos. Já os coeficientes estimados para as exportações de manufaturados dos estados como porcentagem PIB (LEXPORTMANUFATPIB) são negativos, sendo que em um dos dois modelos (modelo 4) ocorreu significância estatística. 


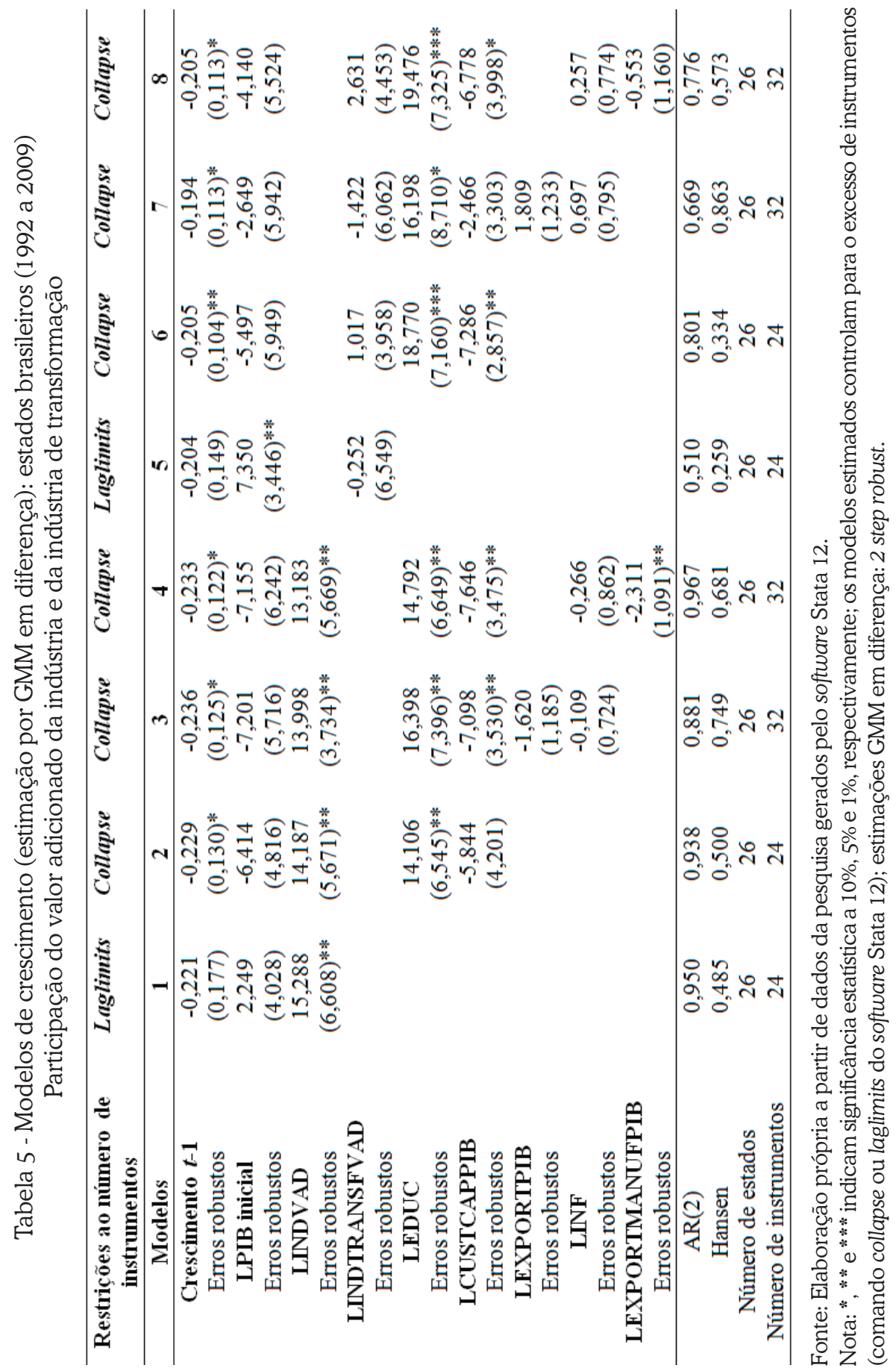


Os testes de ausência de autocorrelação de segunda ordem, AR(2), e de validade dos instrumentos (Hansen) reportados na Tabela 5 indicam não rejeição da hipótese nula em todos os modelos estimados, ou seja, os modelos não possuem autocorrelação de segunda ordem, e o conjunto dos instrumentos é válido.

\section{Considerações Finais}

A literatura econômica, de forma geral, atribui um papel importante da atividade industrial para sustentar o crescimento econômico de longo prazo, na medida em que tal atividade envolve rendimentos crescentes de escala, ganhos de produtividade, efeitos de aprendizado e de transbordamento (spillovers) para com os demais setores da atividade econômica. Nesse sentido, a sustentação e o estímulo ao aumento da participação das atividades industriais na geração de produto, emprego e renda, quando considerada relativamente à participação das atividades intensivas em recursos naturais se mostra fundamental para a obtenção de taxas de crescimento econômico mais robustas, ensejando o uso de políticas industriais ativas, promoção de ganhos de produtividade, incorporação de progresso técnico, entre outros, para se evitar um processo de desindustrialização relativa nas economias, com impactos perversos no crescimento do produto.

Com base em tais argumentos, este trabalho procurou investigar os efeitos da indústria geral e da indústria de transformação sobre o crescimento dos estados brasileiros. Cabe ressaltar que a literatura econômica nesse tema específico é bastante incipiente. Alguns estudos destacam os efeitos da abertura comercial, da composição dos gastos públicos, da educação e das instituições para explicar a taxa de crescimento dos diversos estados brasileiros. Em síntese, percebe-se a importância do grau de abertura comercial, da melhor qualidade dos gastos públicos (infraestrutura, educação, saúde, entre outros, contra gastos de custeio e financeiros), da melhoria do nível educacional, de políticas de redistribuição de renda, da qualidade institucional e da incorporação tecnológica para aumentar a produtividade e, em consequência, ampliar a taxa de crescimento dos PIBs estaduais no Brasil.

Nesse contexto, as evidências econométricas obtidas através da estimação dos modelos de crescimento do PIB real per capita com dados em painel de 1992 a 2009 para os estados brasileiros sugerem algumas lições importantes a ser assimiladas.

A estimação dos modelos de crescimento por EF ou EA indica uma relação direta e estatisticamente significativa entre o PIB da indústria com a taxa de crescimento, sendo que uma variação de 1\% no PIB da indústria tem um impacto médio de $1,78 \%$ ao ano no crescimento. O mesmo papel não pode ser atribuído ao PIB da indústria de transformação, dado que na maioria dos modelos não se verificou sig-

VIEIRA, F. V.; VERISSIMO, M. P.; AVELLAR, A. P. M. Indústria e Crescimento: uma Análise... 
nificância estatística. Há evidência, também, da importância das variáveis médias dos anos de estudo indicando uma relação direta destas com a taxa de crescimento dos estados brasileiros, ou seja, maior (menor) nível de educação está associado a maiores (menores) taxas de crescimento. Observou-se, também, evidência de que os estados que gastam mais (menos) com custeio e capital em média têm menor (maior) taxa de crescimento. As estimações para a variável participação do valor adicionado da indústria revela uma relação direta e significativa com a taxa de crescimento, indicando que uma variação de $1 \%$ na participação da indústria no valor adicionado tem um impacto médio de $1,67 \%$ ao ano na taxa de crescimento dos estados brasileiros. O mesmo papel não pode ser atribuído à participação da indústria de transformação no valor adicionado, dado que na maioria dos modelos não se verificou significância estatística. Foi confirmada a significância estatística dos gastos com custeio e capital, indicando que os estados com maiores (menores) gastos do governo na média são associados a menores (maiores) taxas de crescimento. As estimações revelaram, ainda, que as exportações totais e de manufaturados em relação ao PIB, além da taxa de inflação, não se mostraram estatisticamente significativas para explicar a taxa de crescimento dos estados.

A seção 4 deste trabalho destacou as vantagens dos modelos GMM em relação aos modelos de EF ou EA, vantagens essas associadas à possibilidade de lidar com endogenia das variáveis explicativas - além da inclusão da variável dependente defasada como variável explicativa do crescimento dos estados - e, nesse sentido, incorporar a persistência da taxa de crescimento no tempo. Assim sendo, as lições empíricas do trabalho devem estar atreladas aos resultados sumarizados na sequência.

Os resultados da estimação do modelo de crescimento dos estados brasileiros por GMM em diferença revelam uma relação direta da variável PIB da indústria com a taxa de crescimento indicando que uma variação de $1 \%$ no PIB da indústria (PIB da indústria de transformação) tem um impacto médio de 3,58\% (1,47\%) ao ano na taxa de crescimento. As magnitudes dos coeficientes estimados para o PIB da indústria e da indústria de transformação são maiores nas estimações por GMM em diferença quando comparadas com as estimações por EF ou EA. As estimações dos coeficientes para a variável participação da indústria no valor adicionado revela uma relação direta e significativa com a taxa de crescimento dos estados brasileiros, indicando que uma variação de $1 \%$ na participação da indústria no valor adicionado tem um impacto médio de $4,72 \%$ ao ano na taxa de crescimento. As magnitudes dos coeficientes estimados para a participação da indústria no valor adicionado são maiores nas estimações por GMM em diferença quando comparadas com as estimações por EF ou EA. As estimações revelam, ainda, que não se tem significância estatística para a variável participação da indústria de transformação no valor adicionado. 
As estimações dos modelos de crescimento por GMM em diferença revelam, ainda, a existência de significância estatística para a variável média dos anos de estudo, proxy para capital humano, ressaltando a importância da educação para o crescimento nos estados. Outra variável a ser destacada diz respeito aos gastos públicos com custeio e capital como porcentagem do PIB, cujos coeficientes estimados foram todos negativos, com significância estatística em sete dos doze modelos estimados, associando maiores (menores) gastos com custeio e capital a menores (maiores) taxas de crescimento do PIB. Os coeficientes estimados para as variáveis exportações totais e de manufaturados como porcentagem do PIB, além da taxa de inflação, não se mostraram estatisticamente significativos, com exceção de um dos quatro modelos estimados com as exportações de manufaturados.

A lição empírica geral a ser assimilada, com base nas estimações GMM, ressalta a relevância do PIB da indústria e da indústria de transformação, que são indicadores comparativos do tamanho desses setores entre os diversos estados brasileiros, além da participação do valor adicionado da indústria geral, mas não da participação do valor adicionado da indústria de transformação. Além disso, o nível de escolaridade média tem um impacto positivo, enquanto os gastos com custeio e capital possuem uma relação inversa com o crescimento do PIB dos estados brasileiros no período analisado. Por fim, o desempenho do setor exportador e da inflação nos estados não se mostrou estatisticamente relevante para explicar as diferenças nas taxas de crescimento econômico.

\section{Referências}

ABELLES, M.; RIVAS, D. Growth versus development: different patterns of industrial growth in Latin America during the 'boom' years. Buenos Aires: Cepal, 2010.

ARELLANO, M.; BOND, S. Some tests of specification for panel data: Monte Carlo evidence and an application to employment equations. Review of Economic Studies, v. 58, n. 2, p. 277-97, 1991.

ARELLANO, M.; BOVER, O. Another look at the instrumental variable estimation of errorcomponents models. Journal of Econometrics, v. 68, n. 1, p. 29-51, 1995.

BAER, W. Import substitution and industrialization in Latin America: experiences and interpretations. Latin American Research Review, v. 7, n. 1, p. 95-122, 1972.

BARRO, R. J.; SALA-I-MARTIN, X. Convergence Across States and Regions. Brookings Papers on Economic Activity, vol.1, p.107-182, 1991

BLUNDELL, R; BOND, S. Initial conditions and moment restrictions in dynamic panel data models. Journal of Econometrics, v. 87, n. 1, p. 115-143, 1998.

BRASIL. Ministério da Fazenda. Estatísticas. Brasília, DF: MF, 2013a. Disponível em: < http:// www.fazenda.gov.br/> Acesso em: 01 maio 2013. 
BRASIL. Ministério da Fazenda. Secretaria do Tesouro Nacional. Séries Temporais. Brasília, DF: STN, 2013b. Disponível em: <http:/www3.tesouro.fazenda.gov.br/series_temporais/ principal.aspx\#ancora_consulta>. Acesso em: 01 maio 2013.

. Ministério do Desenvolvimento, Indústria e Comércio Exterior. Balança Comercial Brasileira: Unidades da Federação. Brasília, DF: MDIC, 2013c. Disponível em: < http://www. mdic.gov.br//sitio/interna/interna.php?area $=5 \mathcal{E} 3 \mathrm{menu}=1078 \mathcal{E} \mathrm{refr}=1076>$. Acesso em: 01 maio 2013.

BRESSER-PEREIRA, L. C.; MARCONI, N. Existe doença holandesa no Brasil? In: FÓRUM DE ECONOMIA DA FUNDAÇÃO GETÚLIO VARGAS, 4., 2008, São Paulo. Anais... São Paulo: FGV, 2008. Disponível em: < http://www.bresserpereira.org.br>. Acesso em: 01 out. 2008.

CHENERY, H. B. Patterns of industrial growth. The American Economic Review, v. 50, n. 4, p. 624-654, 1960.

COMISIÓN ECONÓMICA PARA AMÉRICA LATINA Y EL CARIBE. Progreso técnico y cambio estructural en América Latina. Santiago de Chile: Cepal, 2008.

CRUZ, B. O.; SANTOS, I. R. S. Dinâmica do emprego industrial no Brasil entre 1990 a 2009: uma visão regional da desindustrialização. Rio de Janeiro: Instituto de Pesquisa Econômica Aplicada, nov. 2011. (Texto para Discussão, n. 1673).

DE LONG, J. B.; SUMMERS, L. H. Equipment investment and economic growth. Quarterly Journal of Economics, v. 106, n. 2, p. 445-502, 1991.

DIAS, J.; DIAS, M. H. Crescimento econômico e as políticas de distribuição de renda e investimento em educação nos estados brasileiros: teoria e análise econométrica. Estudos Econômicos, São Paulo, v. 37, n. 4, p. 701-743, 2007.

FERREIRA, P. C. G.; ELLERY JÚNIOR, R. G. Convergência entre a renda per capita dos estados brasileiros. Revista de Econometria, v. 16, n. 1, p. 83-103, 1996.

HIRSCHMAN, A. O. The strategy of economic development. New Haven: Yale University Press, 1958.

IBGE. Contas Regionais do Brasil. Rio de Janeiro, 2013b. Disponível em: < http://www.ibge. gov.br/home/estatistica/pesquisas/pesquisa_resultados.php?id_pesquisa $=5>$. Acesso em: 01 maio 2013.

Contas Regionais do Brasil. Rio de Janeiro: IBGE, 2008. (Série Relatório Metodológicos n. 37). Disponível em: < http://www.ibge.gov.br/home/estatistica/pesquisas/ pesquisa_resultados.php?id_pesquisa =5>. Acesso em: 01 maio 2013.

. Pesquisa industrial mensal: produção física por estados. Rio de Janeiro: IBGE, 2013a. Disponível em: <http://www.sidra.ibge.gov.br/bda/acervo/acervo2. $\mathrm{asp} ? \mathrm{e}=\mathrm{c} \mathcal{E} \mathrm{p}=\mathrm{PZE} \mathcal{E} \mathrm{v}=28 \mathcal{E} \mathrm{z}=\mathrm{t} \mathcal{E} \mathrm{O}=22>$ Acesso em: 01 maio 2013.

IPEADATA. Banco de Dados do Instituto de Pesquisa em Economia Aplicada. Brasília: Ipea, 2013. Disponível em: <http://www.ipeadata.gov.br> Acesso em: 01 maio 2013. 
LIBÂNIO, G.; MORO, S. Manufacturing industry and economic growth in Latin America: a Kaldorian approach. In: ENCONTRO NACIONAL DE ECONOMIA, 37., 2009, Foz do Iguaçu. Anais... Foz do Iguaçu: Anpec, 2009. Disponível em: < http://www.anpec.org.br/ encontro2009/inscricao.on/arquivos/000-98e6915698ae97aca03d8e866339ae4e.pdf > . Acesso em: 01 jan. 2012.

MENEZES-FILHO, N. et al. Instituições e diferenças de renda entre os estados brasileiros: uma análise histórica. In: ENCONTRO NACIONAL DE ECONOMIA, 34., 2006, Salvador. Anais... Salvador: Anpec, 2006.

OREIRO, J. L.; FEIJÓ, C. A. Desindustrialização: conceituação, causas, efeitos e o caso brasileiro. Revista de Economia Política, v. 30, n. 2, p.219-232, abr.-jun. 2010.

PIEPER, U. Deindustrialization and the social and economic sustainability nexus in developing countries: cross-country evidence on productivity and employment. New York: Center For Economic Policy Analysis, May 1998. (Working Paper, 10). p. 1-47.

ROCHA, F.; GIUBERTI, A. C. Composição do Gasto Público e Crescimento Econômico: uma avaliação macroeconômica da qualidade dos gastos dos estados brasileiros. Revista Economia Aplicada, v. 11, n. 4, p. 463-485, 2007.

RODRIK, D. Growth after the Crisis. Cambridge, 2009. Disponível em: <http://www. growthcommission.org/storage/cgdev/documents/financial_crisis/rodrikafterthecrisis.pdf > Acesso em: 30 jan. 2012.

ROODMAN, D. A note on the theme of too many instruments. Oxford Bulletin of Economics and Statistics, v. 71, n. 1, p.135-158, 2009b.

. How to do xtabond2: An introduction to difference and system GMM in Stata. Stata Journal, v. 9, n. 1, p. 86-136, 2009a.

SAMPAIO, D. P.; PEREIRA, V. V. Doença holandesa no brasil: uma sugestão de análise conceitual comparada. In: ENCONTRO NACIONAL DE ECONOMIA POLÍTICA, 14., 2009, São Paulo, Anais... São Paulo: SEP, 2009.

SOUZA, N. J. Abertura Comercial e Crescimento dos Estados Brasileiros, 1991/2000. Revista Teoria e Evidência Econômica, Passo Fundo, v. 11, n. 21, p. 41-61, 2003.

TREGENNA, F. Characterising deindustrialization: an analysis of changes in manufacturing employment and output internationally. Cambridge Journal of Economics, v. 33, n. 3, p. 433-466, Nov. 2008.

WINDMEIJER, F. A Finite Sample Correction for the Variance of Linear Efficient Two-step GMM Estimators. Journal of Econometrics, v. 126, n.1, p. 25-51, May 2005. 


\section{Apêndice A - Definição e Fonte das Variáveis}

Quadro 1 - Lista de variáveis, definições e fontes

\begin{tabular}{|c|c|c|}
\hline Variável & Definição & Fonte \\
\hline Crescimento & $\begin{array}{l}\text { Taxa de crescimento do PIB } \\
\text { real per capita em reais de } 2000 \\
\text { (porcentagem. }\end{array}$ & IBGE \\
\hline LPIBINICIAL & $\begin{array}{l}\text { Logaritmo natural do PIB inicial per } \\
\text { capita em R\$ de 2000-1992, 1995, } \\
1998,2001,2004,2007 .\end{array}$ & IBGE \\
\hline LEDUC & $\begin{array}{l}\text { Anos de estudo - média - pessoas } 25 \\
\text { anos e mais. }\end{array}$ & Ipea \\
\hline LINF & $\begin{array}{l}\text { Logaritmo natural da taxa de in- } \\
\text { flação medida pelo Índice Nacional } \\
\text { de Preços ao Consumidor. }\end{array}$ & IBGE \\
\hline LCUSTCAPPIB & $\begin{array}{l}\text { Logaritmo natural das despesas } \\
\text { de capital e custeio estadual: } \\
\text { porcentagem do PIB. }\end{array}$ & $\begin{array}{l}\text { Ministério } \\
\text { da Fazenda } \\
\text { e Secretaria } \\
\text { do Tesouro } \\
\text { Nacional. }\end{array}$ \\
\hline LPIBIND & $\begin{array}{l}\text { Logaritmo natural do PIB da indús- } \\
\text { tria a preços básicos: reais mil. }\end{array}$ & IBGE \\
\hline LPIBINDTRANSF & $\begin{array}{l}\text { Logaritmo natural do PIB da in- } \\
\text { dústria de transformação a preços } \\
\text { básicos: reais mil. }\end{array}$ & IBGE \\
\hline LINDVAD & $\begin{array}{l}\text { Participação da indústria no valor } \\
\text { adicionado bruto a preços básicos. }\end{array}$ & IBGE \\
\hline LINDTRANSFVAD & $\begin{array}{l}\text { Participação da indústria de trans- } \\
\text { formação no valor adicionado bruto } \\
\text { a preços básicos. }\end{array}$ & IBGE \\
\hline LEXPORTPIB & $\begin{array}{l}\text { Logaritmo natural das exportações } \\
\text { totais dos estados (porcentagem do } \\
\text { PIB). }\end{array}$ & MDIC \\
\hline LEXPORTMANUFPIB & $\begin{array}{l}\text { Logaritmo natural das exportações } \\
\text { de manufaturados dos estados (por- } \\
\text { centagem do PIB). }\end{array}$ & MDIC \\
\hline $\begin{array}{l}\text { yr9294, yr9597, } \\
\text { yr982000, yr20012003, } \\
\text { yr20042006, } \\
\text { yr20072009 }\end{array}$ & Dummies temporais. & \\
\hline
\end{tabular}

Fonte: Elaboração própria. 
Recebido em: 11/12/13.

Aceito em: 19/08/14. 